\title{
Produtividade de feijão no sistema plantio direto com aplicação de calcário e zinco
}

\author{
Nand Kumar Fageria( ${ }^{(1)}$ e Luís Fernando Stone ${ }^{(1)}$ \\ (1)Embrapa Arroz e Feijão, Caixa Postal 179, CEP 75375-000 Santo Antônio de Goiás, GO. E-mail: fageria@cnpaf.embrapa.br, \\ stone@cnpaf.embrapa.br
}

\begin{abstract}
Resumo - A acidez dos solos de cerrado é um dos fatores mais limitantes na produtividade das culturas, e a deficiência de micronutrientes desses solos está relacionada com o aumento do pH. O objetivo deste trabalho foi avaliar o efeito da aplicação de calcário e Zn na produtividade do feijão, em Latossolo Vermelho distrófico típico. O experimento foi realizado no campo durante três anos consecutivos. As doses de calcário aplicadas foram 0,12 e $24 \mathrm{Mg} \mathrm{ha}^{-1}$ e de $\mathrm{Zn}, 0,5,10,20,40$ e $80 \mathrm{~kg} \mathrm{ha}^{-1}$. Houve aumento significativo na produtividade do feijão com a aplicação de calcário, porém não houve resposta à aplicação de zinco. Foram estabelecidos valores adequados de algumas propriedades químicas do solo para a cultura do feijoeiro. Considerando a média de duas profundi-

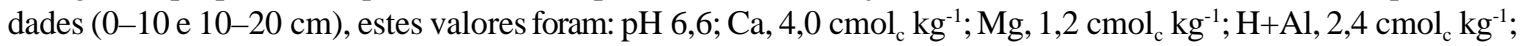
saturação por acidez, 33,9\%; CTC, 7,9 $\mathrm{cmol}_{\mathrm{c}} \mathrm{kg}^{-1}$; saturação por bases, $69,3 \%$, saturação por $\mathrm{Ca}, 46,8 \%$; saturação por $\mathrm{Mg}, 15,3 \%$; saturação por $\mathrm{K}, 4,2 \%$; relação $\mathrm{Ca} / \mathrm{Mg}, 4,6$; relação $\mathrm{Ca} / \mathrm{K}, 19,1$ e relação $\mathrm{Mg} / \mathrm{K}, 6,7$.
\end{abstract}

Termos para indexação: Phaseolus vulgaris, grão, rendimento, propriedade química do solo.

\section{Yield of common bean in no-tillage system with application of lime and zinc}

\begin{abstract}
Soil acidity is one of the most limiting factors to yield in crop production in cerrado soils and micronutrientes deficiency is associated to increase in $\mathrm{pH}$ of cerrado soil with liming. The objective of this study was to evaluate effects of liming and $\mathrm{Zn}$ application to a distrofic Dark-Red Latosol (Typic Haplorthox) on bean yield. A field experiment was conducted during three consecutive years. Lime rates used were 0, 12, and $24 \mathrm{Mg} \mathrm{ha}^{-1}$, and $\mathrm{Zn}$ rates were $0,5,10,20,40$, and $80 \mathrm{~kg} \mathrm{ha}^{-1}$. Bean yield was significantly increased with the application of lime, however, $\mathrm{Zn}$ treatment did not present the same performance. There was a significant change in soil chemical properties at 0-10 and 10-20 cm depths with the application of lime. Values for maximum grain yield were $\mathrm{pH}$ 6.6; $\mathrm{Ca}, 4.0 \mathrm{cmol}_{\mathrm{c}} \mathrm{kg}^{-1} ; \mathrm{Mg}, 1.2 \mathrm{cmol}_{\mathrm{c}} \mathrm{kg}^{-1} ; \mathrm{H}+\mathrm{Al}, 2.4 \mathrm{cmol}_{\mathrm{c}} \mathrm{kg}^{-1}$; acidity saturation, 33.9\%; CEC, $7.9 \mathrm{cmol}_{\mathrm{c}} \mathrm{kg}^{-1}$; base saturation, $69.3 \%$, Ca saturation, $46.8 \%$; $\mathrm{Mg}$ saturation, $15.3 \% ; \mathrm{K}$ saturation, $4.2 \% ; \mathrm{Ca} / \mathrm{Mg}$ ratio 4.6; $\mathrm{Ca} / \mathrm{K}$ ratio 19.1 and $\mathrm{Mg} / \mathrm{K}$ ratio 6.7 across two soil depths.
\end{abstract}

Index terms: Phaseolus vulgaris, grain, yields, soil chemical properties.

\section{Introdução}

A cultura do feijoeiro (Phaseolus vulgaris L.) destaca-se, no Brasil, por sua importância na alimentação da população. O feijoeiro é uma das principais culturas plantadas na entressafra em sistemas irrigados, nas regiões central e sudeste do Brasil (Barbosa Filho et al., 2001). Os solos da região central ou de cerrado são ácidos e apresentam deficiência ou toxidez de alguns elementos, que limitam a produtividade agrícola (Fageria \& Stone, 1999). Além de deficiência e toxidez nutricional, os solos da região de cerrado apresentam baixa capacidade de retenção de água e baixa atividade dos microrganismos. No entanto, com a correção de acidez, é possível transformá-los em solos férteis, ou seja, capazes de proporcionarem produtividades mais elevadas. A calagem ainda é uma das práticas mais baratas e efetivas na correção da acidez do solo. No Brasil, existem vastas reservas de calcário distribuídas em todo o território nacional. A deficiência de $\mathrm{Zn}$ é freqüentemente observada em culturas anuais nos solos de cerrado e está relacionada com o baixo teor natural desse micronutriente no solo e quando são utilizadas doses elevadas de calcário na correção da acidez (Barbosa Filho et al., 1999; Fageria, 2000).

A degradação do solo tem sido uma preocupação constante da comunidade científica, por causa da redução na produtividade das culturas, do aumento no custo de produção e dos danos ao meio ambiente. Uma das práticas mais efetivas e eficientes de conservação do 
solo é o uso do cultivo mínimo ou do plantio direito. A prática de plantio direto reduz a erosão do solo, aumenta a retenção de água do solo, controla a população de plantas daninhas e reduz o custo de produção. Além disso, permite racionalizar os custos, o uso de equipamentos e o tempo, e melhorar a qualidade do solo (Salton et al., 1998). O cultivo mínimo aliado a rotação das culturas promove a acumulação de carbono orgânico no solo e, desta maneira, sequiestra $\mathrm{CO}_{2}$, que seria liberado na atmosfera (West \& Post, 2002).

O sistema de plantio direto é considerado componente importante de sistemas de produção agrícola sustentáveis. Esta prática é utilizada há muito tempo na região Sul do País (Salton et al., 1998). Na Região Central, a área cultivada com o sistema de plantio direto vem aumentando nos últimos anos (Salton et al., 1998), porém são necessárias informações adicionais sobre calagem e adubação com Zn para incrementar o uso dessa prática na produção de feijão, na região do Cerrado.

O objetivo deste trabalho foi avaliar o efeito da aplicação de calcário e $\mathrm{Zn}$ na produtividade do feijão num Latossolo Vermelho distrófico típico.

\section{Material e Métodos}

O experimento foi realizado no campo, durante três anos consecutivos (1999-2001), num Latossolo Vermelho distrófico típico (Oxissolo) na Fazenda Capivara, da Embrapa Arroz e Feijão, localizada no Município de Santo Antônio de Goiás, GO. Os resultados da análise química e granulométrica das amostras do solo da área experimental, coletadas antes da instalação do experimento (Tabela 1), foram realizadas conforme Embrapa (1997).

As doses de calcário utilizadas foram 0,12 e $24 \mathrm{Mg} \mathrm{ha}^{-1}$, aplicadas e incorporadas no solo com grade, cinco meses antes da semeadura do primeiro cultivo de feijão de inverno (última semana de maio a primeira semana de setembro). Estas doses foram determinadas com base no trabalho de Fageria (2001a), que mostrou a produção máxima de feijão com a aplicação de $10 \mathrm{Mg} \mathrm{ha}^{-1} \mathrm{de}$ calcário, quando o teor de argila no solo de cerrado estava em torno de $330 \mathrm{~g} \mathrm{~kg}^{-1}$. As doses de $\mathrm{Zn}$, aplicadas na época da semeadura do primeiro cultivo, a lanço e incorporadas, foram $0,5,12,20,40$ e $80 \mathrm{~kg} \mathrm{ha}^{-1}$, como sulfato de zinco. Estas doses foram definidas com base nos trabalhos de Barbosa Filho et al. (1999) e Fageria et al. (2002), que recomendam de 5 a $30 \mathrm{~kg} \mathrm{ha}^{-1}$, para aplicação no sulco. Na aplicação a lanço, é necessário aproximadamente três vezes mais adubo que na aplicação no sulco. O calcário utilizado possuía PRNT 88,3\%, $\mathrm{CaO}, 32,5 \%$ e $\mathrm{MgO}, 13,3 \%$. O feijão foi cultivado no inverno, com irrigação por pivô central, durante três anos consecutivos; nos dois últimos anos, foi feito o plantio direto. Aplicaram-se $20 \mathrm{~kg} \mathrm{ha}^{-1} \mathrm{de} \mathrm{N}$ (uréia), $120 \mathrm{~kg} \mathrm{ha}^{-1}$ de $\mathrm{P}_{2} \mathrm{O}_{5}$ (superfosfato triplo) e $60 \mathrm{~kg} \mathrm{ha}^{-1}$ de $\mathrm{K}_{2} \mathrm{O}$ (cloreto de potássio) por ocasião da semeadura. Foram aplicados $50 \mathrm{~kg} \mathrm{ha}^{-1}$ de $\mathrm{N}$ (uréia) em cobertura aos $27 \mathrm{e}$ 41 dias após o plantio. A mesma adubação básica foi repetida nos anos subseqüentes. $\mathrm{O}$ delineamento experimental foi o de blocos ao acaso, com doses de calcário nas parcelas principais e doses de Zn nas subparcelas, com três repetições. A cultivar utilizada foi a Pérola, nos três cultivos. O espaçamento foi de $40 \mathrm{~cm}$ entre fileiras, com 19 sementes por metro. O tamanho das parcelas foi de $42 \times 42 \mathrm{~m}$ e das subparcelas, de $7 \times 7 \mathrm{~m}$. Entre parcelas o espaçamento foi de $2 \mathrm{~m}$ e entre subparcelas, de $1 \mathrm{~m}$. As plantas daninhas foram controladas com a aplicação do herbicida pós-emergente. Colheram-se as sete fileiras centrais com $4 \mathrm{~m}$ cada uma para determinação da produtividade. Após a colheita, coletaram-se amostras de solo em cada parcela, compostas por 50 subamostras, a $0-10$ e $10-20 \mathrm{~cm}$ de profundidade, para análise química. Foram calculadas a capacidade de troca de cátions (CTC), a saturação por bases, a saturação por acidez e as relações entre $\mathrm{Ca}, \mathrm{Mg}$ e $\mathrm{K}$ e a CTC.

Tabela 1. Características químicas e granulométricas em duas profundidades do Latossolo Vermelho distrófico típico da área experimental antes da aplicação dos tratamentos, em Santo Antônio de Goiás, GO.

\begin{tabular}{lcc}
\hline Propriedades do solo & $0-10 \mathrm{~cm}$ & $10-20 \mathrm{~cm}$ \\
\hline $\mathrm{pH}$ em água & 5,7 & 5,6 \\
$\mathrm{Ca}\left(\mathrm{cmol}_{\mathrm{c}} \mathrm{kg}^{-1}\right)$ & 2,1 & 1,4 \\
$\mathrm{Mg}\left(\mathrm{cmol}_{\mathrm{c}} \mathrm{kg}^{-1}\right)$ & 1,2 & 0,9 \\
$\mathrm{Al}\left(\mathrm{cmol}_{\mathrm{c}} \mathrm{kg}^{-1}\right)$ & 0,1 & 0,1 \\
$\mathrm{H}+\mathrm{Al}\left(\mathrm{cmol}_{\mathrm{c}} \mathrm{kg}^{-1}\right)$ & 5,1 & 5,3 \\
$\mathrm{P}\left(\mathrm{mg} \mathrm{kg}^{-1}\right)$ & 175 & 8,2 \\
$\mathrm{~K}\left(\mathrm{mg} \mathrm{kg}^{-1}\right)$ & 87 & 56 \\
$\mathrm{Cu}\left(\mathrm{mg} \mathrm{kg}^{-1}\right)$ & 2,3 & 2,3 \\
$\mathrm{Zn}\left(\mathrm{mg} \mathrm{kg}^{-1}\right)$ & 6,4 & 6,5 \\
$\mathrm{Fe}\left(\mathrm{mg} \mathrm{kg}^{-1}\right)$ & 110 & 110 \\
Mn $\left(\mathrm{mg} \mathrm{kg}^{-1}\right)$ & 14 & 11 \\
Matéria orgânica $\left(\mathrm{g} \mathrm{kg}^{-1}\right)$ & 17 & 15 \\
Argila $\left(\mathrm{g} \mathrm{kg}^{-1}\right)$ & 370 & 360 \\
Silte $\left(\mathrm{g} \mathrm{kg}^{-1}\right)$ & 200 & 215 \\
Areia $\left(\mathrm{g} \mathrm{kg}^{-1}\right)$ & 430 & 425 \\
\hline
\end{tabular}


Os dados foram submetidos à análise de variância e as médias comparadas pelo teste de Tukey a 5\% de probabilidade. Foram ajustadas equações entre as propriedades químicas do solo e a produção de grãos.

\section{Resultados e Discussão}

A interação entre anos de cultivos e calcário não foi significativa em relação à produção, portanto, apenas os dados médios dos três cultivos foram apresentados (Tabela 2). A aplicação de $12 \mathrm{Mg} \mathrm{ha}^{-1}$ de calcário aumentou a produção em $35 \%$ em relação à testemunha. Não houve diferença significativa entre as doses de calcário de 12 e $24 \mathrm{Mg} \mathrm{ha}^{-1}$. O feijoeiro tem apresentado aumento significativo de produtividade com a aplicação de calcário em solo de cerrado (Miranda et al., 1980; Quaggio et al., 1982; Caires et al., 2000; Fageria, 2001a). Fageria (2001a) relatou que a produção máxima de feijão foi obtida com a aplicação de $10 \mathrm{Mg} \mathrm{ha}^{-1}$ de calcário em solo de cerrado, no sistema de preparo convencional de solo. Raij \& Quaggio (1997) determinaram que as doses econômicas de calcário, no solo de cerrado, para o feijão, situaram-se em torno de $7 \mathrm{Mg} \mathrm{ha}^{-1}$ no sistema de preparo convencional de solo. A resposta à maior dose de calcário neste trabalho, em relação aos resultados encontrados anteriormente, pode estar relacionada com o sistema de cultivo diferente e também

Tabela 2. Produtividade do feijoeiro em razão de doses de calcário e zinco aplicadas num solo de cerrado.

\begin{tabular}{lc}
\hline Calcário $\left(\mathrm{Mg} \mathrm{ha}^{-1}\right)$ & Grãos $\left(\mathrm{kg} \mathrm{ha}^{-1}\right)$ \\
\hline 0 & $2.260 \mathrm{~b}$ \\
12 & $3.060 \mathrm{a}$ \\
24 & $2.979 \mathrm{a}$ \\
\hline $\mathrm{Zn}\left(\mathrm{kg} \mathrm{ha}^{-1}\right)$ & \\
0 & 2.771 \\
5 & 2.660 \\
12 & 2.754 \\
20 & 2.806 \\
40 & 2.792 \\
80 & 2.814 \\
\hline Teste F & \\
Cultivo (C) & $* *$ \\
Calcário (CA) & $* *$ \\
CxCA & $\mathrm{ns}$ \\
Zinco (Zn) & $\mathrm{ns}$ \\
CxZn & $\mathrm{ns}$ \\
CxCAxZn & $\mathrm{ns}$ \\
\hline
\end{tabular}

${ }^{(1)}$ Médias de três cultivos seguidas pela mesma letra não diferem entre si

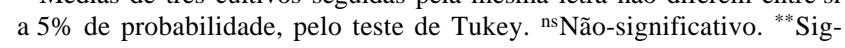
nificativo a $1 \%$ de probabilidade. com o alto teor de argila ( $37 \%$ na camada de $0-10 \mathrm{~cm}$ e $36 \%$ na camada de $10-20 \mathrm{~cm}$ ). Outro fator que pode ter contribuído para a resposta à alta dose de calcário foi o uso de cultivares de feijão com alto potencial de produtividade.

Não houve efeito significativo na produção com a aplicação de $\mathrm{Zn}$ em virtude de seu teor no solo na época da semeadura. O nível crítico de $\mathrm{Zn}$ no solo de cerrado é de $0,7 \mathrm{mg} \mathrm{kg}^{-1}$ para a cultura de feijão, pelo extrator Mehlich 1 (Fageria, 2000) e o seu teor no solo, antes da instalação do experimento, era de $6,4 \mathrm{mg} \mathrm{kg}^{-1} \mathrm{e}$ $6,5 \mathrm{mg} \mathrm{kg}^{-1}$, respectivamente, nas profundidades de $0-10$ e $10-20 \mathrm{~cm}$ (Tabela 1). As pesquisas de micronutrientes em área de cerrado apontam o baixo teor natural no solo e a correção da acidez do solo com calcário como as principais causas de aparecimento de deficiência de micronutrientes, até mesmo do $\mathrm{Zn}$ (Barbosa Filho et al., 1999). O Zn é geralmente mais disponível para as plantas em solos ácidos do que em solos alcalinos (Malavolta, 1980). A acumulação de Zn, Fe e Mn diminui significativamente em solo de cerrado com o aumento de $\mathrm{pH}$, o que pode estar relacionado com a absorção ou precipitação desses micronutrientes (Fageria et al., 2002). Tisdale et al. (1985) relataram que a disponibilidade de $\mathrm{Zn}$ diminui cerca de 100 vezes com o aumento de uma unidade de $\mathrm{pH}$. Porém, no presente caso não houve resposta do feijoeiro à aplicação de $\mathrm{Zn}$, o que mostra que não ocorreu diminuição suficiente deste nutriente no solo para causar a sua deficiência.

A interação entre anos de cultivos e calagem nas propriedades químicas avaliadas foi significativa, dados relativos a essas propriedades nos três anos são apresentados na Tabela 3. Houve mudança significativa nas propriedades químicas do solo com a aplicação de calcário, tanto na camada superficial $(0-10 \mathrm{~cm})$ como no subsolo (10-20 cm) em três anos de cultivo. Porém, os valores foram maiores na camada superficial, com exceção de $\mathrm{H}+\mathrm{Al}$ e saturação por acidez. $\mathrm{O}$ alto $\mathrm{pH}$ na camada superficial foi resultado de altos teores de $\mathrm{Ca}$ e $\mathrm{Mg}$, nesta camada, em comparação com a subcamada $(10-20 \mathrm{~cm})$. Hussain et al. (1999) relataram alto teor de Ca na camada superficial $(0-5 \mathrm{~cm})$ em comparação à camada de $5-15 \mathrm{~cm}$ de profundidade, no sistema de plantio direto. Souza \& Alves (2003) também relataram maior teor de $\mathrm{Ca}, \mathrm{Mg}, \mathrm{P}, \mathrm{K}$ e $\mathrm{pH}$ na camada superficial $(0-10 \mathrm{~cm})$ em comparação com a camada de $10-20 \mathrm{~cm}$ de profundidade. Os valores do $\mathrm{pH}$ para produção máxima de feijão foram de 6,8 na camada superficial e 6,3 na subsuperficial, com valor médio de 6,6 nas duas ca- 
madas (Tabela 4). $\mathrm{O} \mathrm{pH}$ adequado para a cultura do feijoeiro é de 6,2 em solo de cerrado no sistema convencional de plantio (Fageria, 2001b).

A saturação por bases aumentou significativamente com a aplicação de calcário, porém os valores foram maiores na camada superficial do que na subsuperficial $(10-20 \mathrm{~cm})$. Os altos valores na camada superficial também estão relacionados com os maiores teores de $\mathrm{Ca}$ e $\mathrm{Mg}$ nesta camada (Tabela 3). Os altos valores de $\mathrm{Ca}$ e $\mathrm{Mg}$ na camada superficial estão relacionados com a não incorporação de $\mathrm{Ca}$ e $\mathrm{Mg}$ no sistema plantio direto e também com a acumulação de restos culturais na ca- mada superficial. A saturação por bases teve associação significativa com a produção de feijão, com variabilidade de $51 \%$ na camada superficial e $60 \%$ na camada $10-20 \mathrm{~cm}$ de profundidade. A média das duas profundidades mostrou a variabilidade na produção de feijão com saturação por bases de $56 \%$. O valor adequado de saturação por bases foi de $82,2 \%$ na camada superficial e $58,6 \%$ na subsuperficial, com valor médio de $69,3 \%$ nas duas camadas. Os valores adequados de saturação por bases para o feijão, em solo de cerrado, variam de 53\% a $70 \%$ no sistema convencional (Lopes et al., 1991; Fageria 2001b).

Tabela 3. Propriedades químicas do solo após colheita do primeiro, segundo e terceiro cultivos de feijão nos diferentes tratamentos de calcário ${ }^{(1)}$.

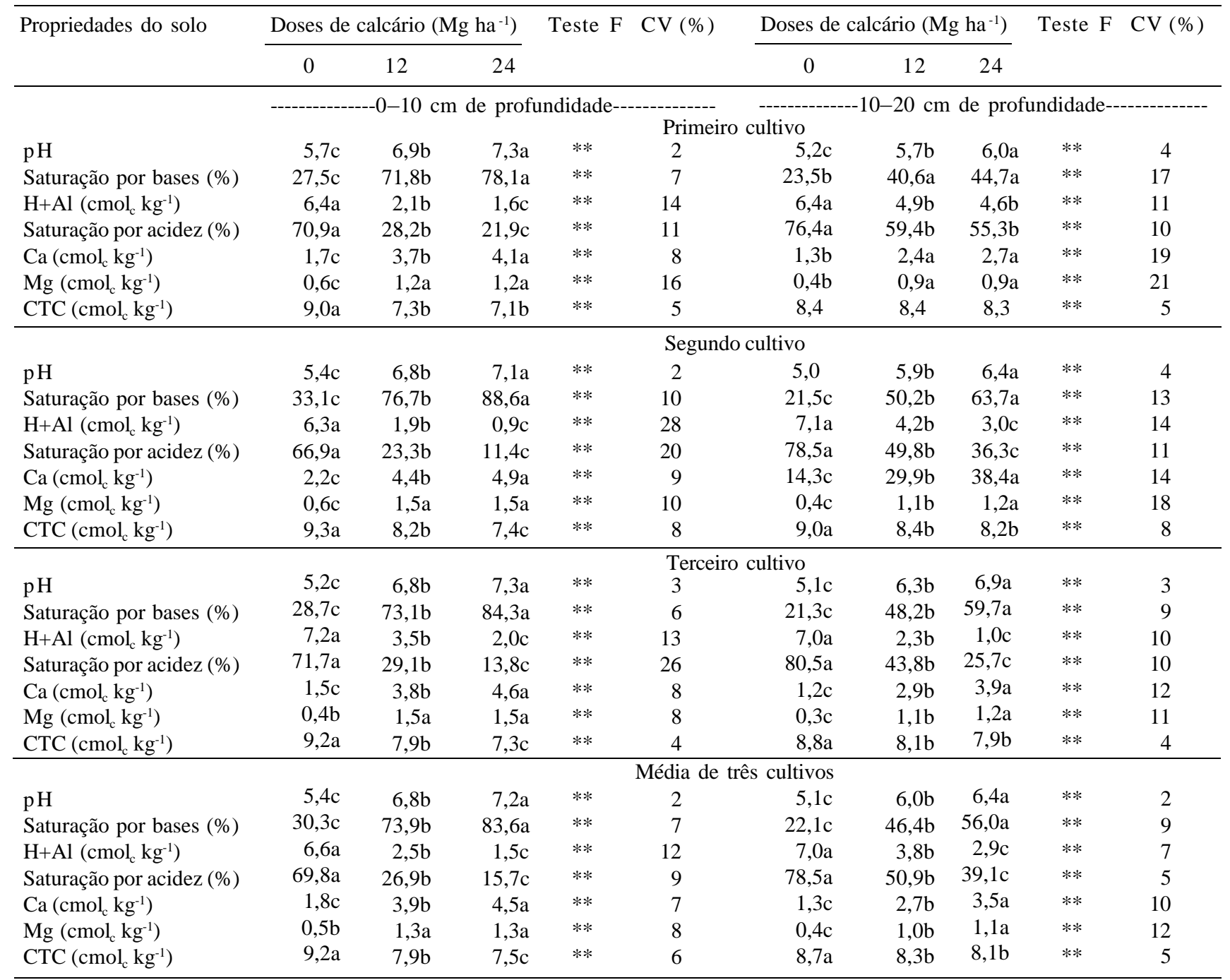

${ }^{(1)}$ Médias seguidas pela mesma letra, na linha, para cada profundidade do solo, não diferem entre si a $5 \%$ de probabilidade, pelo teste de Tukey.

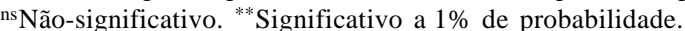


A acidez do solo ( $\mathrm{H}+\mathrm{Al})$ e saturação por acidez variaram significativamente com a aplicação de calcário. Porém, os valores foram maiores na camada subsuperficial $(10-20 \mathrm{~cm})$ do que na camada superficial $(0-10 \mathrm{~cm})$. O teor de $\mathrm{Al}$ foi quase totalmente neutralizado com a aplicação de $12 \mathrm{Mg} \mathrm{ha}^{-1}$ de calcário, mas o
$\mathrm{H}^{+}$remanescente contribuiu para a acidez do solo. Os valores críticos médios de $\mathrm{H}+\mathrm{Al}$ e de saturação por acidez para produção de feijão foram de $2,4 \mathrm{cmol}_{\mathrm{c}} \mathrm{kg}^{-1}$ e $33,9 \%$, respectivamente (Tabela 4). Isto significa que, na presença de valores superiores, pode ocorrer toxidez de $\mathrm{H}+\mathrm{Al}$ para a cultura de feijão em sistema de plantio

Tabela 4. Relação entre propriedades químicas do solo $(\mathrm{X})$ e produção de grãos de feijão (Y). Os valores são a média de três anos de cultivo ${ }^{(1)}$.

\begin{tabular}{|c|c|c|c|}
\hline Propriedades do solo & Equação & $\mathrm{R}^{2}$ & Valor para produção máxima \\
\hline \multicolumn{4}{|c|}{$0-10 \mathrm{~cm}$ de profundidade } \\
\hline $\mathrm{pH}$ & $Y=-14.764,16+5.206,13 X-380,3576 X^{2}$ & $0,5419 * *$ & 6,8 \\
\hline $\mathrm{Ca}\left(\mathrm{cmol}_{\mathrm{c}} \mathrm{kg}^{-1}\right)$ & $Y=1.185,935+707,0167 X-64,8000 X^{2}$ & $0,5329 * *$ & 5,4 \\
\hline $\operatorname{Mg}\left(\mathrm{cmol}_{\mathrm{c}} \mathrm{kg}^{-1}\right)$ & $Y=1.864,4820+595,9188 X+164,5383 X^{2}$ & $0,5718 * *$ & 1,8 \\
\hline $\mathrm{H}+\mathrm{Al}\left(\mathrm{cmol}_{\mathrm{c}} \mathrm{kg}^{-1}\right)$ & $Y=3.008,615+38,4890 X-22,3670 X^{2}$ & $0,5022 * *$ & 0,9 \\
\hline Saturação por acidez $(\%)$ & $Y=2.846,662+15,2307 X-0,3258 X^{2}$ & $0,5221 * *$ & 23,4 \\
\hline $\mathrm{CTC}\left(\mathrm{cmol}_{\mathrm{c}} \mathrm{kg}^{-1}\right)$ & $Y=-13.378,63+4.216,6390 X-271,2248 X^{2}$ & $0,4071 * *$ & 7,8 \\
\hline Saturação por bases (\%) & $Y=1.193,78+44,4125 X-0,2700 X^{2}$ & $0,5140 * *$ & 82,2 \\
\hline Saturação por $\mathrm{Ca}(\%)$ & $Y=1.129,95+67,7427 X-0,6049 X^{2}$ & $0,5115^{* *}$ & 56,0 \\
\hline Saturação por Mg (\%) & $Y=1.606,3780+116,9958 X-2,1940 X^{2}$ & $0,5311 * *$ & 26,7 \\
\hline Saturação por K $(\%)$ & $Y=1.261,269+404,0941 X+35,6798 X^{2}$ & $0,3362 * *$ & 5,7 \\
\hline Relação Ca/Mg & $Y=8.483,796-3.137,6730 X+415,4579 X^{2}$ & $0,1299 *$ & 2,9 \\
\hline Relação $\mathrm{Ca} / \mathrm{K}$ & $Y=228,7047+327,9211 X-9,4592 X^{2}$ & $0,5545^{* *}$ & 17,3 \\
\hline Relação $\mathrm{Mg} / \mathrm{K}$ & $Y=921,2688+691,0032 x-56,5492 X^{2}$ & $0,5111 * *$ & 6,1 \\
\hline \multicolumn{4}{|c|}{$10-20 \mathrm{~cm}$ de profundidade } \\
\hline $\mathrm{pH}$ & $Y=-1.9574,50+7.207,079 X-573,0695 X^{2}$ & $0,5771 * *$ & 6,3 \\
\hline $\mathrm{Ca}\left(\mathrm{cmol}_{\mathrm{c}} \mathrm{kg}^{-1}\right)$ & $Y=747,643+1.406,9570 x-212,9586 X^{2}$ & $0,6073 * *$ & 3,3 \\
\hline $\operatorname{Mg}\left(\mathrm{cmol}_{\mathrm{c}} \mathrm{kg}^{-1}\right)$ & $Y=1.636,989+1.745,0810 X-418,2525 X^{2}$ & $0,6162 * *$ & 2,1 \\
\hline $\mathrm{H}+\mathrm{Al}\left(\mathrm{cmol}_{\mathrm{c}} \mathrm{kg}^{-1}\right)$ & $Y=2.423,059+386,7655 X-59,4702 X^{2}$ & $0,5623 * *$ & 3,3 \\
\hline Saturação por acidez (\%) & $Y=1.896,832+54,2694 X-0,6342 X^{2}$ & $0,5903 * *$ & 42,8 \\
\hline $\mathrm{CTC}\left(\mathrm{cmol}_{\mathrm{c}} \mathrm{kg}^{-1}\right)$ & $Y=51.667,32-11.288,38 X+649,1171 X^{2}$ & $0,0990^{\mathrm{ns}}$ & 8,3 \\
\hline Saturação por bases (\%) & $Y=950,1212+72,8324 X-0,6217 X^{2}$ & $0,6059 * *$ & 58,6 \\
\hline Saturação por $\mathrm{Ca}(\%)$ & $Y=788,2963+116,1053 X-1,4772 X^{2}$ & $0,5987 * *$ & 39,3 \\
\hline Saturação por Mg (\%) & $Y=1.608,729+152,001 X-3,1545 X^{2}$ & $0,6160 * *$ & 24,1 \\
\hline Saturação por K $(\%)$ & $Y=1.983,76+5.847 X-54,2554 X^{2}$ & $0,072^{\text {ns }}$ & 1,8 \\
\hline Relação Ca/Mg & $Y=9.067,547-3.173,3460 X+362,0437 X^{2}$ & $0,4517 * *$ & 2,7 \\
\hline Relação $\mathrm{Ca} / \mathrm{K}$ & $Y=545,0498+230,8463 X-5,2612 X^{2}$ & $0,4949 * *$ & 21,9 \\
\hline Relação $\mathrm{Mg} / \mathrm{K}$ & $Y=1.292,2410+422,9702 X-25,6964 X^{2}$ & $0,4977 * *$ & 8,2 \\
\hline \multicolumn{4}{|c|}{ Média de $0-10$ e $10-20 \mathrm{~cm}$ de profundidade } \\
\hline $\mathrm{pH}$ & $Y=-17.689,98+6.327,2290 X-482,5805 X^{2}$ & $0,5658 * *$ & 6,6 \\
\hline $\mathrm{Ca}\left(\mathrm{cmol}_{\mathrm{c}} \mathrm{kg}^{-1}\right)$ & $\mathrm{Y}=856,3713+1.093,4790 \mathrm{X}-135,8895 \mathrm{X}^{2}$ & $0,5742 * *$ & 4,0 \\
\hline $\mathrm{Mg}\left(\mathrm{cmol}_{\mathrm{c}} \mathrm{kg}^{-1}\right)$ & $\mathrm{Y}=1.747,4990+1.034,2110 \mathrm{X}$ & $0,6037 * *$ & 1,2 \\
\hline $\mathrm{H}+\mathrm{Al}\left(\mathrm{cmol}_{\mathrm{c}} \mathrm{kg}^{-1}\right)$ & $Y=2.782,0960+200,7515 X-40,9768 X^{2}$ & $0,5339 * *$ & 2,4 \\
\hline Saturação por acidez (\%) & $Y=2.503,6720+31,5171 X-0,4643 X^{2}$ & $0,5554 * *$ & 33,9 \\
\hline $\mathrm{CTC}\left(\mathrm{cmol}_{\mathrm{c}} \mathrm{kg}^{-1}\right)$ & $Y=-29.633,12+8.179,4150 X-512,9004 X^{2}$ & $0,3208 * *$ & 7,9 \\
\hline Saturação por bases $(\%)$ & $Y=1.049,9020+57,4026 X-0,4141 X^{2}$ & $0,5557 * *$ & 69,3 \\
\hline Saturação por $\mathrm{Ca}(\%)$ & $Y=927,8372+90,4341 X-0,9669 X^{2}$ & $0,5526^{* *}$ & 46,8 \\
\hline Saturação por Mg (\%) & $\mathrm{Y}=1.812,42+77,9733 \mathrm{X}$ & $0,5745^{* *}$ & 15,3 \\
\hline Saturação por K $(\%)$ & $Y=-373,6017+1.908,5480 X-225,1399 X^{2}$ & $0,3250 * *$ & 4,2 \\
\hline Relação Ca/Mg & $Y=7.861,1270-2.476,7290+269,8845 X^{2}$ & $0,2821 * *$ & 4,6 \\
\hline Relação $\mathrm{Ca} / \mathrm{K}$ & $Y=121,3962+310,9937 X-8,1177 X^{2}$ & $0,5676^{* *}$ & 19,1 \\
\hline Relação $\mathrm{Mg} / \mathrm{K}$ & $Y=917,4143+635,0469 X-47,4527 X^{2}$ & $0,5276^{* *}$ & 6,7 \\
\hline
\end{tabular}

${ }^{(1)}$ Valores para produção máxima foram calculados com base nas equações de regressão em que $\mathrm{R}^{2}$ foi significativo e, nos casos em que $\mathrm{R}^{2}$ não foi significativo e o valor de $\beta_{1}$ foi negativo ou a equação foi linear, o valor médio do tratamento de $12 \mathrm{Mg} \mathrm{ha}^{-1}$ de calcário foi considerado porque a

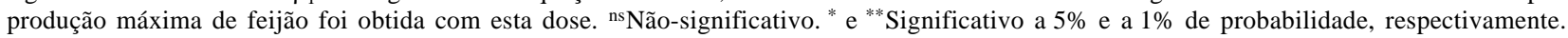


direto em solo de cerrado. Não existem dados para comparação dos valores críticos de $\mathrm{H}+\mathrm{AL}$ e saturação por bases para a cultura de feijão em solo de cerrado no sistema de plantio direto.

A saturação por $\mathrm{Ca}, \mathrm{Mg}$ e $\mathrm{K}$ tiveram associações significativas com a produção de feijão. Os valores médios das duas profundidades para a produção máxima foram de 46,8\% de Ca, $15,3 \%$ de $\mathrm{Mg}$ e $4,2 \%$ de potássio. Os valores adequados destas propriedades químicas do solo relatados para o feijão foram $37 \%$ de $\mathrm{Ca}, 16 \%$ de $\mathrm{Mg}$ e 2,6\% de $\mathrm{K}$ em sistema de plantio convencional (Fageria, 2001b). Isto mostra que os valores de $\mathrm{Ca}$ e $\mathrm{K}$ são altos no sistema de plantio direto para produção de feijão, em comparação com o sistema de plantio convencional. Os valores adequados da relação $\mathrm{Ca} / \mathrm{Mg}, \mathrm{Ca} / \mathrm{K}$ e $\mathrm{Mg} / \mathrm{K}$ foram de 4,6, 19,1 e 6,7 (média das duas profundidades). Cochrane (1989) fez levantamento de alguns solos de cerrado no Distrito Federal, e encontrou valores médios da relação $\mathrm{Ca} / \mathrm{Mg}$ de 0,9 , $\mathrm{Ca} / \mathrm{K}$ de 1,9 e $\mathrm{Mg} / \mathrm{K}$ de 2,3 e considerou que esses valores são muito baixos para as culturas anuais, para se obter produção ótima nesses solos.

\section{Conclusões}

1. A aplicação de calcário até a dose de $12 \mathrm{Mg} \mathrm{ha}^{-1}$ aumenta a produtividade de feijão em solo argiloso de cerrado sob plantio direito.

2. Mesmo com a aplicação de altas doses de calcário, a produtividade de feijão não é afetada pela aplicação de zinco em solo argiloso de cerrado sob plantio direto.

\section{Referências}

BARBOSA FILHO, M.P.; FAGERIA, N.K.; SILVA, O.F. da. Aplicação de nitrogênio em cobertura no feijoeiro irrigado. Santo Antônio de Goiás: Embrapa-CNPAF, 2001. 8p. (Circular Técnica, 49).

BARBOSA FILHO, M.P.; FAGERIA, N.K.; SILVA, O.F. da. Correção de deficiência de micronutrientes em arroz de terras altas. Santo Antônio de Goiás: Embrapa-CNPAF, 1999. 21p. (Documentos, 93).

CAIRES, E.F.; BANZATTO, D.A.; FONSECA, A.F. Calagem na superfície em sistema plantio direto. Revista Brasileira de Ciência do Solo, v. 24, p.161-169, 2000.

COCHRANE, T.T. Chemical properties of native savanna and forest soils in central Brazil. Soil Science Society of America Journal, v.53, p.139-141, 1989.
EMBRAPA. Centro Nacional de Pesquisa de Solos (Rio de Janeiro, RJ). Manual de métodos de análise de solo. 2.ed. Rio de Janeiro, 1997. 212p.

FAGERIA, N.K. Efeito da calagem na produção de arroz, feijão, milho e soja em solo de cerrado. Pesquisa Agropecuária Brasileira, v. 36, p.1419-1424, 2001a.

FAGERIA, N.K. Níveis adequados e tóxicos de zinco na produção de arroz, feijão, milho, soja e trigo em solo de cerrado. Revista Brasileira de Engenharia Agrícola e Ambiental, v.4, p.390-395, 2000.

FAGERIA, N.K. Resposta de arroz de terras altas, feijão, milho e soja à saturação por base em solo de cerrado. Revista Brasileira de Engenharia Agrícola e Ambiental, v.5, p.416-424, 2001 b.

FAGERIA, N.K.; BALIGAR, V.C.; CLARK, R.B. Micronutrients in crop production. Advances in Agronomy, v.77, p. 185-268, 2002.

FAGERIA, N.K.; STONE, L.F. Manejo da acidez dos solos de cerrado e de várzea do Brasil. Santo Antônio de Goiás: EmbrapaCNPAF, 1999. 42p. (Documentos, 92).

HUSSAIN, I.; OLSON, K.R.; EBELHAR, S.A. Long-term tillage effects on soil chemical properties and organic matter fractions. Soil Science Society of America Journal, v.63, p.1335-1341, 1999.

LOPES, A.S.; SILVA, M.C.; GUILHERME, L.R.G. Acidez do solo e calagem. São Paulo: Associação Nacional para Difusão de Adubos e Corretivos Agrícolas, 1991. 15p. (Boletim Técnico, 1).

MALAVOLTA, E. Elementos de nutrição mineral de plantas. São Paulo: Agronônica Ceres, 1980. 251p.

MIRANDA, L.N.; MIELNICZUK, J.; LOBATO, E. Calagem e adubação corretiva. In: MARCHETTI, D.; MACHADO, A.D. (Ed.). Cerrado: uso e manejo. Brasília: Editerra, 1980. p.523-591.

QUAGGIO, J.A.; MASCARENHAS, H.A.A.; BATAGLIA, O.C. Resposta da soja à aplicação de doses crescentes de calcário em Latossolo Roxo distrófico de cerrado - II: efeito residual. Revista Brasileira de Ciência do Solo, v.6, p.113-118, 1982.

RAIJ, B. van; QUAGGIO, J.A. Methods used for diagnosis and correction of soil acidity in Brazil: overview. In: INTERNATIONAL SYMPOSIUM ON PLANT-SOIL INTERACTIONS AT LOW $\mathrm{pH}$, 4., 1996, Belo Horizonte. Proceedings. Campinas: Sociedade Brasileira de Ciência do Solo, 1997. p.205-214.

SALTON, J.C.; HERNANI, L.; FONTES, C.Z. Sistema plantio direto: o produtor pergunta, a Embrapa responde. Brasília: Embra pa-SPI; Embrapa-CPAO, 1998. 248p.

SOUZA, Z.M.; ALVES, M.C. Propriedades químicas de um latossolo vermelho distrófico de cerrado sob diferentes usos e manejos. Revista Brasileira de Ciência do Solo, v.27, p.133-139, 2003.

TISDALE, S.L.; NELSON, W.L.; BEATON, J.D. Soil fertility and fertilizers. $4^{\text {th }}$ ed. New York: Macmillan, 1985. 754p.

WEST, T.O.; POST, W.M. Soil organic carbon sequestration rates by tillage and crop rotation: a global data analysis. Soil Science Society of America Journal, v.66, p.1930-1946, 2002.

Recebido em 7 de julho de 2003 e aprovado em 12 de novembro de 2003 
